\title{
Benchmarking of Electro-Optic Monitors for Femtosecond Electron Bunches
}

\author{
G. Berden, ${ }^{1}$ W. A. Gillespie, ${ }^{2}$ S. P. Jamison, ${ }^{3}$ E.-A. Knabbe, ${ }^{4}$ A. M. MacLeod, ${ }^{5}$ A. F. G. van der Meer, ${ }^{1}$ P. J. Phillips,${ }^{2}$ \\ H. Schlarb, ${ }^{4}$ B. Schmidt, ${ }^{4}$ P. Schmüser ${ }^{4}$ and B. Steffen ${ }^{4}$ \\ ${ }^{1}$ FOM Institute for Plasma Physics “Rijnhuizen,” Edisonbaan 14, 3439 MN Nieuwegein, The Netherlands \\ ${ }^{2}$ University of Dundee, Dundee DD1 4HN, United Kingdom \\ ${ }^{3}$ Accelerator Science and Technology Center, STFC Daresbury Laboratory, Warrington, Cheshire WA4 4AD, United Kingdom \\ ${ }^{4}$ Deutsches Elektronen-Synchrotron DESY, Notkestrasse 85, 22603 Hamburg, Germany \\ ${ }^{5}$ School of Computing and Creative Technologies, University of Abertay Dundee, Bell Street, Dundee DD1 1HG, United Kingdom
}

(Received 26 June 2007; published 17 October 2007)

\begin{abstract}
The longitudinal profiles of ultrashort relativistic electron bunches at the soft x-ray free-electron laser FLASH have been investigated using two single-shot detection schemes: an electro-optic (EO) detector measuring the Coulomb field of the bunch and a radio-frequency structure transforming the charge distribution into a transverse streak. A comparison permits an absolute calibration of the EO technique. EO signals as short as $60 \mathrm{fs}$ (rms) have been observed, which is a new record in the EO detection of single electron bunches and close to the limit given by the EO material properties.
\end{abstract}

Intense relativistic electron bunches with a duration of 100 femtoseconds or less are essential for free-electron lasers (FELs) based on the principle of self-amplified spontaneous emission (SASE), such as the ultraviolet and soft x-ray Free-electron LASer at Hamburg (FLASH) [1], and future $x$-ray FELs like the Linac Coherent Light Source [2] at SLAC and the European XFEL [3]. Ultrashort relativistic electron bunches are also produced in plasma wakefield accelerators which have made impressive progress in the last years, see [4-6] and the references quoted therein. Precise knowledge of the temporal profile of the electron bunches is essential for a detailed understanding of the physical processes in all these accelerators $[1,4]$. Two of the most important current techniques for the single-shot direct visualization of longitudinal electron bunch profiles are transverse-deflecting structures (TDS) and electro-optic (EO) detection.

The principle of the TDS was demonstrated in 1964 [7]: the temporal profile of the electron bunch charge density is transferred to a transverse streak on a view screen by a rapidly varying electromagnetic field, analogous to the sawtooth voltage in a conventional oscilloscope tube. The time resolution of the TDS installed at FLASH can reach $15 \mathrm{fs}$ (rms) if the beam optics is optimized to yield the smallest possible beam spot on the view screen [8]. With the optics tuned for FEL operation, the resolution is about 20 fs. Thus, the diagnostic itself is part of the accelerator optics design. The TDS must be several meters long to achieve sufficient streak length, preventing its use in a plasma wakefield accelerator. Furthermore, it is inherently destructive, prohibiting the characterization of bunches that are needed further downstream.

The EO effect has been used extensively in terahertz time domain spectroscopy for over a decade [9], but its exploitation in electron bunch diagnostics is more recent. The need for an absolute temporal signal, rather than just a relative change, which is sufficient in spectroscopic applications, is a uniquely demanding requirement of EO bunch diagnostics. Single-shot EO measurements of picosecond electron bunches were first demonstrated in 2002 [10]. Several variants of EO bunch diagnostics have been applied [10-12], all sharing the underlying principle of utilizing the field-induced birefringence in an electro-optic crystal to convert the time profile of a bunch into a temporal, spectral, or spatial intensity modulation of a probe laser pulse. The EO techniques have the advantage of being nondestructive, thereby permitting correlation studies of EO measurements on selected bunches with the FEL pulses produced by the same bunches. Moreover, the EO signals can serve as arrival time triggers $[11,12]$.

The drawback of the EO method is that it relies on nonlinear optical processes for converting the electric field profile of a bunch into an optical signal; confidence in the understanding of these processes for very short bunches is crucial to the interpretation of the data. The highly desirable calibration of the EO detection process against known ultrashort $\mathrm{THz}$ pulses has not been possible up to now due to the lack of suitable $\mathrm{THz}$ sources. Indeed, the generation of fast $\mathrm{THz}$ pulses is usually accomplished through optical rectification in electro-optic crystals [9], a process which is complementary to EO detection, and as such subject to the identical calibration uncertainties. In this Letter, we present a novel method to overcome this limitation: first the ultrashort electron bunch is precisely characterized by the TDS, and then its transient electric field is used as a known $\mathrm{THz}$ pulse for calibration and validation of the $\mathrm{EO}$ detector.

Most of the measurements reported here were carried out during FEL user operation of FLASH at an electron energy of $450 \mathrm{MeV}$. Time profiles of single electron bunches were obtained without disturbing the quality of the FEL beam. We compare EO and TDS data with the aim 
of investigating the faithfulness and limitations of EO temporal diagnostics for ultrashort bunches. An online time calibration procedure of the EO detector is described that is not affected by bunch-to-bunch timing jitter. Finally, we demonstrate the application of the EO technique to accelerator diagnostics.

At FLASH, electron bunches with an energy of $4 \mathrm{MeV}$, a charge of $0.5-1 \mathrm{nC}$, and a length of 4-5 ps (rms) are produced in a laser-driven photo injector and accelerated to $450-700 \mathrm{MeV}$ by a superconducting linear accelerator (linac). The bunches come in pulse trains (repetition rate $1-10 \mathrm{~Hz}$ ), where each train consists of 1-800 bunches with a spacing of $1 \mu \mathrm{s}$. The SASE process requires high peak currents of several 1000 A which are obtained by reducing the bunch length by nearly 2 orders of magnitude. Longitudinal bunch compression is achieved in two steps. First, an energy slope is imprinted on the bunch by off-crest acceleration in the first 8 radio-frequency (rf) cavities, the particles at the head of the bunch receiving a smaller energy gain than those at the tail. Then, the beam passes through two magnetic chicanes where the trailing electrons travel a shorter distance than the leading ones and are enabled to catch up with them. Because of the cosine shape of the $1.3 \mathrm{GHz}$ rf wave adding a nonlinear term to the position-energy relationship inside the bunch, and to coherent synchrotron radiation effects in the magnetic chicanes, the final bunches consist of a leading spike with an rms width of $<50 \mathrm{fs}$ and a tail extending over several ps. The longitudinal charge density profile of the compressed electron bunches can be measured with a TDS and an EO setup located downstream of the last accelerating section. Finally, the beam generates FEL radiation in a $27 \mathrm{~m}$ long undulator magnet at wavelengths as short as $13 \mathrm{~nm}$.

The TDS was built at SLAC and has been installed and commissioned in FLASH at DESY in collaboration with SLAC [8]. It is a $3.6 \mathrm{~m}$ long traveling wave structure operated at $2.856 \mathrm{GHz}$ in which a transverse electromagnetic field exerts a time-dependent transverse force on the electrons. The bunches pass through the TDS near zero crossing of the rf field and receive no net deflection; however, they are streaked in the vertical direction. Time calibration is achieved by measuring the net deflection for rf phase settings different from zero. Since the fill-time of the TDS is less than a microsecond, a single bunch out of a train can be streaked. With a fast kicker magnet, this bunch is deflected horizontally towards an optical transition radiation (OTR) screen viewed by a CCD camera. The other bunches in the train are not affected. Owing to space constraints, the EO experiment had to be mounted downstream of the TDS. Thus, it is not possible to observe the same electron bunch with both detection schemes since the streaked bunch is too diffuse to produce a good EO signal. In the experiments described here, TDS and EO measurements were carried out on adjacent bunches in the same pulse train.
Electro-optic temporal decoding (EOTD) [11,13] was used since it offers the best time resolution of the singleshot EO detection methods. The titanium-sapphire laser amplifier (pulse length 30 fs FWHM, central wavelength $795 \mathrm{~nm}$, pulse energy $0.5 \mathrm{~mJ}$, repetition rate $1 \mathrm{kHz}$ ) is synchronized to the $1.3 \mathrm{GHz}$ accelerator rf. The laser beam is guided through a $20 \mathrm{~m}$-long optical beam line into the accelerator tunnel where it is split into two beams: the probe beam and the gate beam. The probe beam is stretched to $20 \mathrm{ps}$ with a grating-pair optical stretcher and guided through a polarizer to set the correct polarization with respect to the optical axis of the EO crystal. It is then injected into the linac vacuum chamber at an angle of $6^{\circ}$ with respect to the electron beam and passes through the $65 \mu \mathrm{m}$ thick electro-optic gallium phosphide (GaP) crystal placed at a distance of $4-5 \mathrm{~mm}$ from the electron beam. The birefringence induced by the Coulomb field of the passing electron bunch is translated into a time-dependent elliptical polarization of the stretched probe beam. This in turn is converted into an intensity modulation with a second polarizer located outside the beampipe. The transmittance through this polarizer is minimized in the absence of an electron bunch, and a quarter wave plate is used to remove any residual birefringence. Measurements can either be done in the crossed-polarizer detection scheme [11], yielding an EO signal being proportional to the square of the bunch electric field, $E_{b}^{2}(t)$, or at a polarizer angle a few degrees away from orthogonal polarization. In the latter case, the background is larger, but the EO signal is linear in $E_{b}(t)$. The EO-induced intensity modulation of the probe pulse is measured through cross-correlation of probe and gate pulse in a second-harmonic generation (SHG) crystal. By overlapping the two pulses noncollinearly, a spatially dependent time delay is introduced between the pulses so that when the SHG light is imaged on an intensified CCD camera, the temporal profile of the electron bunch may be derived from the light intensity as a function of position.

An example of a single-shot EOTD measurement during FEL operation, performed at $1^{\circ}$ away from orthogonal polarization, is compared with the TDS measurement of the next bunch in Fig. 1. The EOTD and TDS signals agree in their main features, but the superior time resolution of the TDS technique is evident. The TDS signal exhibits a structure consisting of a narrow peak (rms width $\sigma<$ $30 \mathrm{fs}$ ) and a shoulder, while the electric field pulse $E_{b}(t)$ as measured by EOTD shows only a single peak with $\sigma=60$ fs.

An approximate time calibration of the EOTD setup can be derived from the known laser beam geometry in the cross correlator [11]. We have devised an online calibration procedure with much higher accuracy: just before the cross correlator, the gate pulse is duplicated by inserting two $50 \%$ beam splitters and a motorized delay line into the gate beam path. As a result, the probe pulse will be cross 


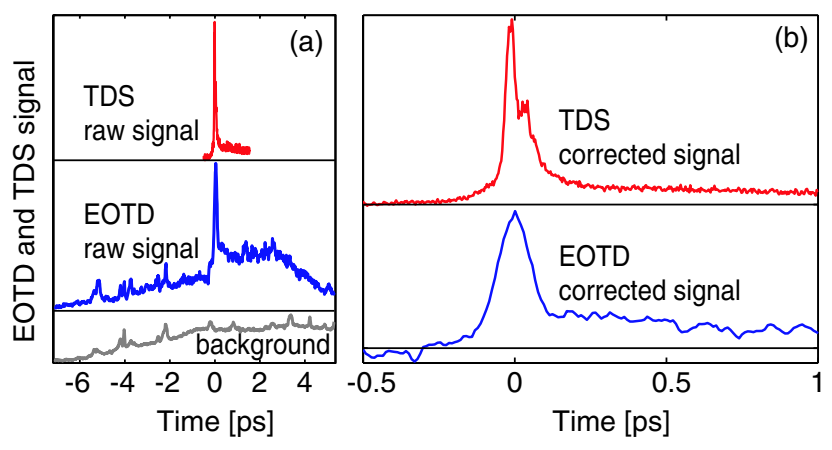

FIG. 1 (color online). Longitudinal bunch charge profile measured during FEL operation with the transverse-deflecting structure (TDS), compared to the electric bunch field $E_{b}(t)$ obtained by single-shot electro-optic temporal decoding (EOTD). The bunch head is at the left side. The full time window is 2 ps for the TDS and 12 ps for EOTD. (a) Raw data. For the EOTD data, the background measured without electron beam is also shown. (b) The normalized TDS and EOTD signals, with backgrounds subtracted, are shown on an expanded time scale.

correlated twice within several picoseconds, and the electron bunch will appear twice in the CCD image, as shown in Fig. 2. Variation of the delay $\Delta T$ between the two gate pulses provides a time calibration with fs accuracy which is not affected by arrival time jitter of the electron bunches.

To address the faithfulness and the limitations of the EOTD technique, we need to consider the response of the $\mathrm{GaP}$ crystal to ultrashort pulses. When the relativistic bunch passes within a few $\mathrm{mm}$ of the EO crystal, the transient electric field is equivalent to a half-cycle $\mathrm{THz}$ pulse impinging on the crystal. The most severe limitation on the resolving power is given by excitation of transverse optical (TO) lattice vibrations in the EO crystal. The lowest TO frequency is $11 \mathrm{THz}$ in GaP which is why we chose it as an EO material in preference to the more common $\mathrm{ZnTe}$ with a lowest TO frequency of $5.3 \mathrm{THz}$. In the vicinity of the resonance, the complex refractive index $n_{1}(f)+i n_{2}(f)$ and the electro-optic coefficient $r_{41}(f)$, determining the size of the EO signal, exhibit strong frequency dependencies. Severe pulse broadening and shape distortions will occur if the incident $\mathrm{THz}$ pulse has significant Fourier components near the $11 \mathrm{THz}$ resonance, which is the

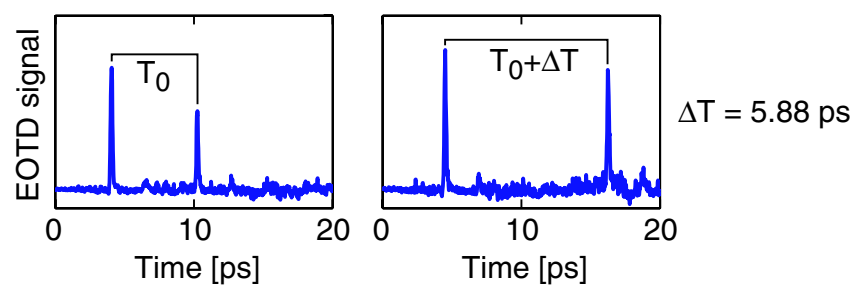

FIG. 2 (color online). Time calibration of the electro-optic measurements is obtained by duplicating the gate pulse (see text). The base delay $T_{0}$ is not precisely known, but the additional delay $\Delta T$ can be varied with fs accuracy. case for 30 fs pulses. Fourier components above $11 \mathrm{THz}$ are strongly suppressed in the $65 \mu \mathrm{m}$-thick GaP crystal used in our EOTD measurements due to group and phase velocity mismatch between $\mathrm{THz}$ pulse and optical laser pulse.

We have simulated the response of a $65 \mu \mathrm{m} \mathrm{GaP}$ crystal to ultrashort electron bunches as characterized by the TDS. The steps are indicated in Fig. 3(a). The equivalent $\mathrm{THz}$ pulse entering the EO crystal, which is identical to the Coulomb field of the electron bunch at a distance of $4.5 \mathrm{~mm}$ from the electron beam, has an estimated rms width of $40 \mathrm{fs}$. This pulse has been taken as input for a numerical simulation of the electro-optic process in $\mathrm{GaP}$ [14], using published material data. The Fourier components of the $\mathrm{THz}$ pulse are multiplied by the complex electro-optic response function $G(f)$ of the $\mathrm{GaP}$ crystal and the EO coefficient $r_{41}(f)$, and then the phase retardation between the two orthogonal polarization components of the laser beam is calculated. The computed EO signal has $\sigma \approx 55 \mathrm{fs}$. Note that this widening is entirely due to the $11 \mathrm{THz}$ resonance. For the $65 \mu \mathrm{m} \mathrm{GaP}$ crystal, the cutoff arising from the frequency dependence of the EO coefficient $r_{41}$ is lower than that from phase matching, and limits the usable frequency range to $f<8 \mathrm{THz}$.

Figure 3(b) shows that the shape of the measured EOTD signal agrees very well with the predicted one except for a slight increase in width (60 fs), due to the resolution of the optical cross correlator ( $20 \mathrm{fs}$ rms), and a higher intensity in the tail of the bunch. The tail is enhanced by wakefields generated upstream of the EO crystal. Such wakefields are invisible in the TDS measurement because the TDS streaks the electric charge of the bunch and is totally insensitive to electromagnetic fields traveling down the beam pipe. The almost perfect agreement in the width of the leading spike
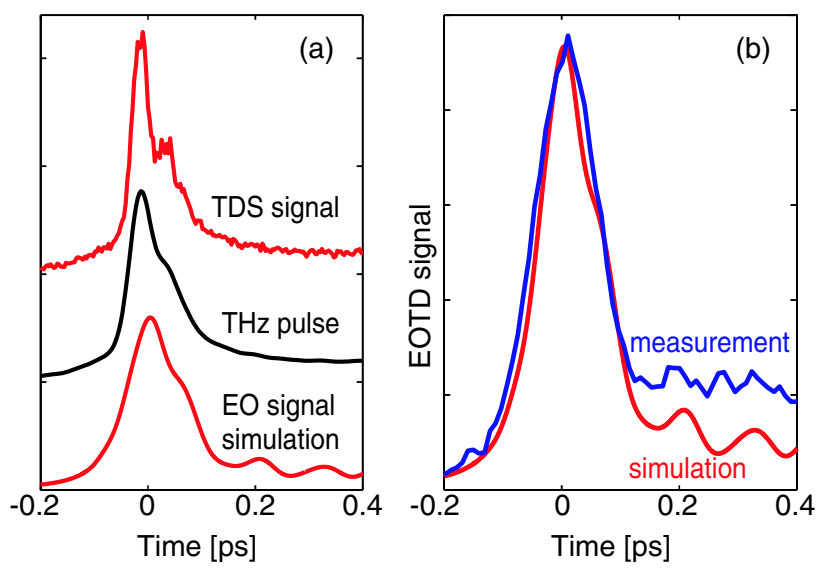

FIG. 3 (color online). Steps in the comparison between TDS and EOTD signal shapes. (a) From top to bottom: TDS signal, equivalent $\mathrm{THz}$ pulse at EO crystal, simulated EO signal. (b) Comparison between simulation and EOTD measurement. The simulated and the measured signals have been scaled to the same peak height. 

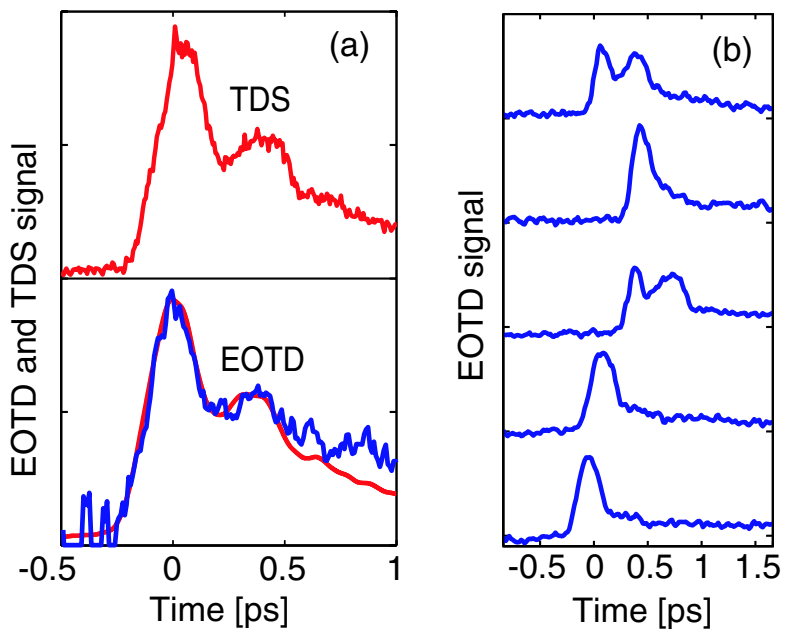

FIG. 4 (color online). (a) TDS and EOTD signals of bunches showing a double-peak structure due to a detuned compression system. The simulated EO signal is shown by the smooth curve in the lower panel. (b) Sequence of EOTD signals obtained with the machine tuned for minimum bunch length and the bunch compression feedback switched off, resulting in shot-to-shot fluctuations in bunch shape.

between measurement and prediction means that we have basically reached the resolution limit for EO bunch shape reconstruction with GaP. Electron bunches with an rms length in the $30 \mathrm{fs}$ range appear thus stretched to about 55 fs. Note, however, that longer electron bunches $(\sigma \geq$ $90 \mathrm{fs}$ ) will be faithfully reconstructed by EOTD because these pulses contain negligible Fourier components in the resonance region of GaP. To demonstrate this experimentally, the rf phase in the bunch compression system was detuned on purpose from its optimal value to generate wider bunches with a double-peak structure. For these wide bunches, the agreement between TDS and EOTD is indeed very good as shown in Fig. 4(a). The simulated EO signal agrees very well with the measured EO signal except in the tail, which can be attributed to wakefield effects.

We remark that an improved EO time resolution might be expected from new EO polymers with a broader frequency response than $\mathrm{GaP}$ [15], but these polymers are not yet stable enough to be used in accelerators.

The EO measurements are nondestructive and thus ideally suited for online monitoring of the accelerator performance. To illustrate this application, the machine was tuned for minimum bunch length, and the bunch compression feedback system of FLASH was switched off, resulting in shot-to-shot bunch shape fluctuations which are clearly visible in the EOTD data [Fig. 4(b)]. The EO system thus provides an excellent diagnostic for the proper functioning of the bunch compression system.

In a separate experiment, the capabilities of an EO system to deliver start signals, corresponding to peak beam current, for pump-probe experiments at the FEL, were studied. Bunch arrival time measurements were carried out with the simpler electro-optic spectral decoding technique [10] which needs only a laser oscillator. The oscillator can be more accurately synchronized to the accelerator $\mathrm{rf}$ than the amplifier required for EOTD. An rms accuracy of the arrival time measurement of $65 \mathrm{fs}$ was reached that was dominated by timing jitter in the synchronization system between laser and rf. This jitter will be reduced to about 20 fs by the future fiber-laser-based synchronization and timing system of FLASH [16].

In summary, nondestructive, single-shot, longitudinal electron bunch profile detectors based on electro-optic detection with femtosecond lasers have been successfully implemented and calibrated at a soft $\mathrm{x}$-ray FEL. The best time resolution ever in the electro-optic analysis of ultrashort electron bunches has been achieved, and an absolute calibration of the EO process has been made possible by using incident pulses of well-known shape that were characterized by a transverse-deflecting structure (TDS) of excellent time resolution. Although the EO technique is inferior to the TDS in terms of time resolution, it has the considerable advantage of being nondestructive and does allow trigger signals for pump-probe experiments at the FEL to be provided. Moreover, the EO setup covers a larger time window, yields valuable information on wakefield effects, and requires much less space in the linac than the TDS.

The assistance of Professor D. Jaroszynski and the support of the RCUK alpha-X program and FOM/NWO is gratefully acknowledged.

[1] V. Ayvazyan et al., Eur. Phys. J. D 37, 297 (2006).

[2] http://www-ssrl.slac.stanford.edu/lcls/.

[3] http://www.xfel.net.

[4] J. van Tilborg et al., Phys. Rev. Lett. 96, 014801 (2006).

[5] W. P. Leemans et al., Nature Phys. 2, 696 (2006).

[6] J. Faure et al., Nature (London) 444, 737 (2006).

[7] O.H. Altenmueller, R. R. Larsen, and G. A. Loew, Rev. Sci. Instrum. 35, 438 (1964).

[8] M. Hüning et al., in Proceedings of the 27th International Free Electron Laser Conference (SLAC, Stanford, 2005), p. 538.

[9] B. Ferguson and X.-C. Zhang, Nat. Mater. 1, 26 (2002); C. A. Schmuttenmaer, Chem. Rev. 104, 1759 (2004), and references therein.

[10] I. Wilke et al., Phys. Rev. Lett. 88, 124801 (2002).

[11] G. Berden et al., Phys. Rev. Lett. 93, 114802 (2004).

[12] A. L. Cavalieri et al., Phys. Rev. Lett. 94, 114801 (2005).

[13] S. P. Jamison et al., Opt. Lett. 28, 1710 (2003).

[14] S. Casalbuoni et al., TESLA Report No. 2005-01, 2005.

[15] X. Zheng, A. Sinyukov, and L. M. Hayden, Appl. Phys. Lett. 87, 081115 (2005).

[16] A. Winter et al., in Proceedings of EPAC 2006, (EPS-AG, Edinburgh, UK, 2006), p. 1063. 